\title{
Multi-Objective Optimization of a Wing Fence on an Unmanned Aerial Vehicle using Surrogate-Derived Gradients
}

\author{
Jolan Wauters · Ivo Couckuyt • Nicolas \\ Knudde • Tom Dhaene • Joris Degroote
}

Received: date / Accepted: date

\begin{abstract}
In this paper the multi-objective, multi-fidelity optimization of a wing fence on an unmanned aerial vehicle (UAV) near stall is presented. The UAV under consideration is characterized by a blended wing body (BWB), which increases its efficiency, and a tailless design, which leads to a swept wing to ensure longitudinal static stability. The consequence is a possible appearance of a nose-up moment, loss of lift initiating at the tips and reduced controllability during landing, commonly referred to as tip-stall. A possible solution to counter this phenomenon is wing fences: planes placed on top of the wing aligned with the flow and developed from the idea of stopping the transverse component of the boundary layer flow. These are optimized to obtain the design that would fence off the appearance of a pitch-up moment at high angles of attack, without a significant loss of lift and controllability. This brings forth a constrained multi-objective optimization problem. The evaluations are performed through Unsteady Reynolds-Averaged Navier-Stokes (URANS) simulations. However, since controllability cannot be directly assessed through Computational Fluid Dynamics (CFD), surrogate-derived gra-

Conducted as part of the SBO research project 140068 EUFORIA (Efficient Uncertainty quantification For Optimization in Robust design of Industrial Applications) under the financial support of the IWT, the Flemish agency of Innovation through Science and Technology. This work was carried out using the STEVIN Supercomputer Infrastructure at Ghent University, funded by Ghent University, the Flemish Supercomputer Center (VSC), the Hercules Foundation and the Flemish Government - department EWI.
\end{abstract}

J. Wauters, J.Degroote

Ghent University

Department of Flow, Heat and Combustion Mechanics

Sint-Pietersnieuwstraat 41, 9000 Gent, Belgium

E-mail: \{jolan.wauters, joris.degroote\}@ugent.be

I. Couckuyt, N. Knudde, D. Dhaene

Ghent University

Department of Information Technology (INTEC)

iGent, Technologiepark-Zwijnaarde 15, 9052 Gent, Belgium

E-mail: \{ivo.couckuyt, nicolas.knudde, tom.dhaene\}@ugent.be 
dients are used. An efficient global optimization framework is developed employing surrogate modeling, namely regressive universal co-Kriging, updated using a multi-objective formulation of the expected improvement. The result is a wing fence design that extends the flight envelope of the aircraft, obtained with a feasible computational budget.

Keywords Multi-Objective Optimization · Surrogate Based Optimization · Regressive Universal Co-Kringing · Tip stall · Wing Fence · Unmanned Aerial Vehicle

\section{Introduction}

The widespread use of unmanned aerial vehicles (UAV) has become clear over recent years. Within the range of UAVs that exists nowadays, this paper focuses on those that operate at a chord-based Reynolds number $\left(R e_{c}\right)$ below $5 \times 10^{5}$, the condition which is referred to as low Reynolds number flow [18].

Airfoils operating at low Reynolds number conditions are characterized by the appearance of a transitional separation bubble [35]. It is therefore of importance to correctly resolve this phenomenon to assure a correct estimation of the flight behavior of the UAV. This can be obtained by means of computational fluid dynamics (CFD) if appropriate turbulence modeling is applied. The relatively low computational cost that is attributed to Reynolds-Averaged Navier-Stokes (RANS) simulations allows its use in increasingly complex 3D geometries. In the last couple of decades, a number of turbulence models have been developed that attempt to model the transition phenomena that are attributed to low Reynolds number flow. Here Menter et al.'s $\gamma-R e_{\theta}$ model [22] is used based on a comparative study of transitional turbulence models [36]. It results in deviations of CL and CD up to $10 \%$ for the investigated conditions near the stall angle.

The unmanned aerial vehicle which will serve as the base of this study can be noted by its blended wing body (BWB) design [26]: a tailless aircraft with its fuselage, the aircraft's main body, integrated in the wing. The absence of a horizontal tailplane enforces the sweeping and twisting of the wing to obtain longitudinal static stability, the intrinsic desire of the airplane to correct minor changes in its angle of attack (AoA). However, the sweeping of the wings introduces a pressure gradient on the wing normal to the free stream, decreasing from the root to the tip. When the air is moving relatively slowly, such as in the boundary layer, the flow will be influenced by this distribution and be sucked towards the tip. Moving towards the tip, this effect will result in a more rapid growth of the thickness of the boundary layer and in a higher likelihood of flow separation at high angles of attack, for example during landing. This leads to a loss of lift, commonly referred to as stall, and loss of effectiveness of the control surfaces (elevons). A special case of stall is tip-stall. As its name indicates, the tips of the wing start stalling first. This is a common but unwanted characteristic of swept wings. Since the tips are 
generally located behind the center of gravity (CoG), tip stalling will result in a pitch-up moment, thus pulling the plane further into stall.

A number of solutions have been developped to tackle the problem of tip stalling, such as vortex generators [24,1] and wing fences [37]. The wing fence, boundary layer fence or potential fence can be defined as a plate which is placed on top of the wing aligned with air flow and, depending on its design, extending up to the trailing edge or extending over the leading edge to the lower surface. It was the first aerodynamic device introduced on swept wings to tackle the phenomenon of tip stalling. It has a straightforward concept: stopping the transverse component of the boundary layer flow and changing the lift distribution $[9,25,31]$. It is unambiguous to install on the wing without making any modifications to the shape of the wing itself, making it an attractive device.

Optimization of the geometry of the wing fence to obtain a stable, but as slow as possible descent without a severe loss of lift and controllability and in the absence of a nose-up pitching moment translates itself in a constrained multi-objective optimization problem. In the 90s Multi-Objective Evolutionary Algorithms (MOEAs) such as NSGA-ii were often the first choice to tackle such a problem. However, even with the advent of high performance computing (HPC), this can be computational infeasible when the calculation of objectives and constraints relies on CFD simulations. Thus, an efficient framework must be used such as the efficient global optimization (EGO) algorithm by Jones et al. [13], which relies on the introduction of an intermediate level in the form of a surrogate model namely Kriging, which builds forth on the concepts of Gaussian Processes. The EGO algorithm makes use of the Expected Improvement (EI) to update the surrogate, but was developed for single-objective problems. In the previous decade, a series of multi-objective reformulations of the Expected Improvement such as Keane's Euclidean Expected Improvement have been formulated $[17,40,12,14,28,2]$.

The description of the initial geometry and the optimization problem, the definition of the objective functions and constraints and the parameterization of the wing fence are found in $\S 2$. The methodology by which the wing fence is to be optimized is presented in $\S 3$ : a surrogate based optimization framework is proposed combining low-fidelity data obtained on a coarse grid and highfidelity data obtained on a fine grid by URANS simulations using the $\gamma-\operatorname{Re}_{\theta}$ model. Furthermore, the operation conditions are added as variables that can change continuously. As such, we overcome the limitations of multipoint problems where the objective is optimized in a discrete set of operating conditions $[24,16]$. In $\S 4$ the convergence of the optimization is discussed, as well as the initial design and the Pareto front from an aerodynamic point of view. 


\section{Problem Description}

\subsection{Optimization Case}

The minimization of the descent speed in a controllable fashion without the risk of tip-stalling through the addition of a well-positioned and well-designed wing fence translates into a constrained multi-objective optimization case. The calculation of these objectives and constraints will be explained in this section.

In figure 1 , the force diagram of the UAV during descent is presented, where $L$ corresponds with the lift, $D$ with the drag and $W$ with the drone's weight. The latter is a fixed value, while the former two can be written as $C_{L}(\alpha, \delta, R e) \frac{1}{2} \rho V^{2} S$ and $C_{D}(\alpha, \delta, R e) \frac{1}{2} \rho V^{2} S$. In these equations, $C_{L}$ and $C_{D}$ correspond with respectively the lift and drag coefficient, both of which are a function of the angle of attack $\alpha$, the elevon deflection $\delta$ and the Reynolds number Re. $\rho$ corresponds with the density of air, $V$ with the descent speed and $S$ with the projected area of the wing. The Reynolds number is defined as $R e=\rho \cdot V \cdot M A C / \mu$ with $\mu$ the dynamic viscosity of air and $M A C$ is the mean aerodynamic chord defined as $\frac{2}{S} \int_{0}^{b / 2} c(y)^{2} d y$ where $y$ is the coordinate along the span and $b$ is the span. Also present in the diagram are the thrust $T$, the flight path angle $\theta$ and the pitching moment $M_{\text {pitch }}$ around the UAV's CoG.

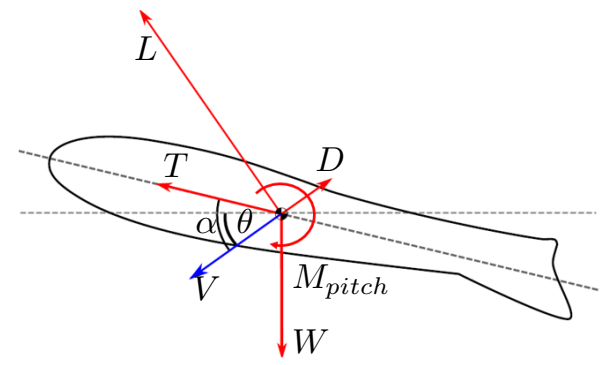

Fig. 1: Force diagram UAV drawn around the CoG

The equilibrium of vertical force, horizontal force and longitudinal moment ${ }^{1}$ are given by equations 1 . Considering the significant influence of the elevon setting on the aerodynamic coefficients, $C_{L}, C_{D}$ and $C_{M, p i t c h}$, the three equations are directly coupled. This set of three non-linear equations contains five unknowns: $\alpha, \delta, \theta, V$ and $T$. Thus, for every $\alpha, \delta$ and $\theta$, the first objective $V$ (and a corresponding $T$ ) can be determined using equations $1 \mathrm{a}$ and $1 \mathrm{~b}$.

\footnotetext{
1 The longitudinal moment is addressed as the pitching moment and defined as the total moment around the tranverse/lateral axis, perpendicular to the symmetry plane with its origin in the center of gravity. For equilibrium flight, this moment must equal zero. In this regard, the pitching moment coefficient of the UAV differs from the conventional pitching moment of an airfoil, which is defined around its aerodynamic center.
} 
However, as three instead of only two out of the five unknowns have been fixed for three equations, they are not independent and have to satisfy the moment equilibrium in equation $1 \mathrm{c}$ which is the first constraint.

Increasing $\theta$, thus descending steeper, decreases $T$ up to the point that a reverse trust must be generated. The influence of $\theta$ on the $V$ is minimal, the latter changing only a few percent when changing the former between $0^{\circ}$ and $20^{\circ}$. In this work, $C_{L}, C_{D}$ and $C_{M, p i t c h}$ are obtained using CFD-simulations, as will be explained further.

$$
\left\{\begin{array}{l}
L \cos (\theta)+D \sin (\theta)+T \sin (\alpha-\theta)=W \\
T \cos (\alpha-\theta)+L \sin (\theta)=D \cos (\theta) \\
M_{p i t c h}=0
\end{array}\right.
$$

The second objective, controllability, is expressed as the roll moment that is obtained through an infinitesimal elevon deflection around the equilibrium position:

$$
M^{\prime}\left(\boldsymbol{x}^{\prime}\right)=\frac{\partial M_{\text {roll }}}{\partial \delta}=\frac{1}{2} \rho V\left(\boldsymbol{x}^{\prime}\right)^{2} S c \frac{\partial C_{M, \text { roll }}\left(\boldsymbol{x}^{\prime}\right)}{\partial \delta}
$$

The absence of tip-stalling corresponds to the absence of the nose-up pitching moment and thus longitudinal static stability, expressed as $\partial C_{M, p i t c h} / \partial \alpha<$ 0 . The longitudinal static stability is added as a second constraint. Thus a constrained multi-objective optimization problem is obtained: the fence design is modified to minimize $V$ while maximizing controllability, subject to moment equilibrium and longitudinal static stability constraints.

$$
\boldsymbol{x}^{\prime}=\arg \min _{\boldsymbol{x}^{\prime}}\left\{V\left(\boldsymbol{x}^{\prime}\right),-M^{\prime}\left(\boldsymbol{x}^{\prime}\right)\right\} \text { s.t. }\left\{\begin{array}{l}
\text { Equations 1a,b,c } \\
\partial C_{M, p i t c h}\left(\boldsymbol{x}^{\prime}\right) / \partial \alpha<0
\end{array}\right.
$$

\subsection{Parameterization}

The parameters of the wing fence design are chosen such that every combination can easily be meshed in a structured manner as to avoid altering the grid discretization error (see figure 2). The fence is defined as the wall between structured mesh blocks, leading to a zero-thickness fence. It is believed by the authors that the inclusion of the thickness of the fence leads to a larger displacement effect. However, the effect of the aforementioned is of lesser impact than for example position and height, which makes its inclusion undeserving taking further into account the additional computational cost attributed to the increased mesh complexity. 


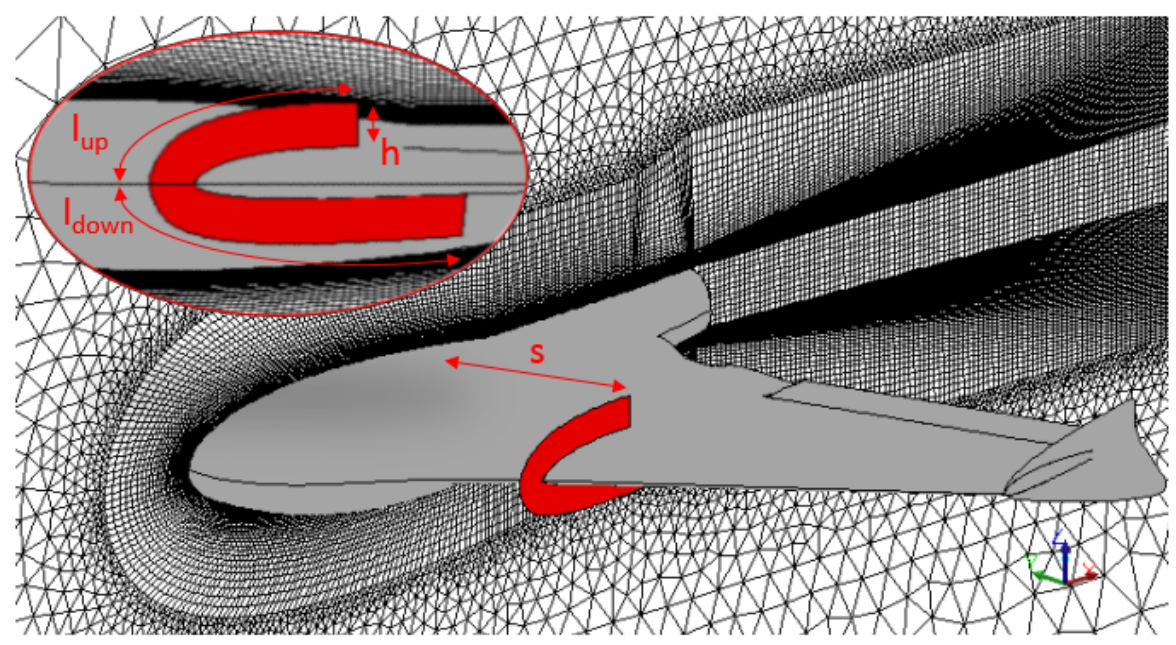

Fig. 2: Mesh detail and parametrization of the wing fence

1. The spanwise position of the fence $(s)$, ranging from the fuselage to the tip.

2. The height of the fence perpendicular to the wing $(h)$, expressed as a percentage of the aerodynamic chord, ranging from 0 to $10 \%$.

3. The length on the suction side of the wing $\left(l_{u p}\right)$, measured from the leading edge to the hinge point of the elevon, expressed as a percentage of the constrained local chord, ranging from 0 to 100\%. The length does not extend to the trailing edge, because it was found that this introduces a strong buffeting on the elevon [27].

4. The length on the pressure side of the wing $\left(l_{\text {down }}\right)$, measured from the leading edge to the hinge point of the elevon, expressed as a percentage of the constrained local chord, ranging from 0 to $100 \%$.

\section{Methodology}

\subsection{Computational Fluid Dynamics}

To model the transitional flow over the UAV we use the correlation-based $\gamma-R e_{\theta}$ model, which builds on the $k-\omega$ SST model, but distinguishes itself through the addition of a supplementary transport equation for the intermittency $\gamma$ and the momentum thickness Reynolds number at transition $R e_{\theta t}$. The former represents the time fraction the flow is turbulent and allows transition to be spread in space. The latter assures that the model captures strong variations of the turbulent intensity, that may occur due to turbulence decay, the influence of the free-stream and the pressure gradient [22]. This model was chosen over other transitional models based on a comperative study with experimental data for high angle of attack behavior [36]. 
All calculations are performed using the CFD-code ANSYS Fluent 16.2 with a second-order upwind discretization for convective terms, second-order central for diffusive terms, least squares cell based gradient approximation, a transient second-order implicit formulation and the SIMPLE pressure-velocity coupling.

The mesh is created in a hybrid manner: a structured hexahedral mesh is created in the close proximity of the body and extending in the wake through the generation of blocks. Defining a wall between these blocks allows the creation of the wing fences. Outside of the structured region an unstructured grid is created composed of tetrahedral cells. Roache's grid convergence index (GCI) is used to quantify the discretization error, corresponding to an estimate of the relative error that would be obtained in case of grid doubling with a second-order method, even if the former was not performed during the study [29].

The refinement study has been performed on three levels for the residual convergence, time-step size and mesh size. In the case of the meshes, two of them make up the low (1.5 million cells) and high (11.5 million cells) fidelity levels used during the optimization. One finer mesh has been added (24 million cells) to establish whether the low and high fidelity mesh lie within the asymptotic range such that the mesh is fine enough to resolve the physical phenomena correctly enough, even on the low fidelity mesh. The meshes have been assessed for the clean geometry at $\alpha=15^{\circ}$, which approximately corresponds to the stall angle. The functional that is evaluated is the lift coefficient, $C_{L}$. The low fidelity calculation with a time step size of $1 \times 10^{-5}$ has a GCI of $18.8 \%$ and takes approximately 500 core hours to finish, while the high fidelity simulation has a GCI of $2.7 \%$ using the same settings and takes approximately 5000 core hours to finish.

\subsection{Surrogate Modeling}

Expensive high fidelity simulations are needed to correctly resolve the progression of separation on the surface of the UAV in the vicinity of the stall angle. However, confronted with the staggering computational cost of a single evaluation, an efficient methodology is sought to find the optimal set of design parameters. An established methodology to answer the problem at hand is found in the field of surrogate modeling, which is actively used for aerospace optimization problems $[38,4,32]$. This implies that, after defining the objective function and the design space, a design of experiments (DoE) is set up to select samples in the design space, for which the objective function is subsequently calculated and of which a surrogate is defined. This cheap to evaluate surrogate or meta-model can subsequently be sampled to define the entire characteristics in function of the geometric design variables.

For the DoE we use a Latin-Hypercube Sampling (LHS) approach [21] and make use of Morris and Mitchell's maximin distance $\phi_{q}$ to quantify the space-filling property, optimized by simulated annealing [23]. 
The surrogate model used is Kriging, which can be seen as the sum of a trend function and Gaussian process: $Y(\boldsymbol{x})=\boldsymbol{f}(\boldsymbol{x})^{T} \boldsymbol{\beta}+Z(\boldsymbol{x})$ with $\mathbb{E}[Z(\boldsymbol{x})]=0$ and $\boldsymbol{f}(\boldsymbol{x})=\left[f_{i}(\boldsymbol{x}), i=1, \ldots, m\right]$ the vector of basis functions, $\boldsymbol{\beta}$ the vector of coefficients and $Z(\boldsymbol{x})$ a Gaussian process $\mathcal{G P}\left(0, \operatorname{cov}\left(\mathbf{y}^{(i)}, \mathbf{y}^{(j)}\right)\right)$, with zero mean and fully described by the covariance function $\operatorname{cov}\left(\mathbf{y}^{(i)}, \mathbf{y}^{(j)}\right)=$ $\sigma^{2} \operatorname{cor}\left(\mathbf{y}^{(i)}, \mathbf{y}^{(j)}\right)$, where $\sigma$ is the process variance and $\operatorname{cor}\left(\mathbf{y}^{(i)}, \mathbf{y}^{(j)}\right)$ is the correlation function between two objectives and is noted for being a function of their inputs and typically written as $\psi\left(\boldsymbol{x}^{i}, \boldsymbol{x}^{j}\right)$. Here the Matérn covariance function is used with $\nu=3 / 2$. The trend is typically the solution of a regression problem and the Gaussian process captures the variation on this trend to exactly interpolate the evaluated data.

In order to determine the parameters of the covariance function, typically referred to as hyperparameters, we maximize the likelihood, $\boldsymbol{L}$, that given the aforementioned surrogate can reproduce the evaluated data [34]. Solving the maximum likelihood estimation (MLE) problem, we can define the Best Linear Unbiased Prediction (BLUP), which allows the prediction of unsampled locations $\boldsymbol{x}^{\prime}$ with respectively the predicted mean and predicted variance:

$$
\begin{aligned}
\mu\left(\boldsymbol{x}^{\prime}\right)= & \boldsymbol{f}\left(\boldsymbol{x}^{\prime}\right)^{T} \boldsymbol{\beta}+\boldsymbol{\psi}\left(\boldsymbol{x}^{\prime}\right)^{T} \boldsymbol{\Psi}^{-1}(\boldsymbol{y}-\mathbf{F} \boldsymbol{\beta}) \\
s^{2}\left(\boldsymbol{x}^{\prime}\right)= & \sigma^{2}\left(1-\boldsymbol{\psi}\left(\boldsymbol{x}^{\prime}\right)^{T} \boldsymbol{\Psi}^{-1} \boldsymbol{\psi}(\mathbf{x})+\left(\mathbf{F}^{T} \boldsymbol{\Psi}^{-1} \boldsymbol{\psi}\left(\boldsymbol{x}^{\prime}\right)-\boldsymbol{f}\left(\boldsymbol{x}^{\prime}\right)\right)^{T}\right. \\
& \left.\left(\mathbf{F}^{T} \boldsymbol{\Psi}^{-1} \mathbf{F}\right)^{-1}\left(\mathbf{F}^{T} \boldsymbol{\Psi}^{-1} \boldsymbol{\psi}\left(\boldsymbol{x}^{\prime}\right)-\boldsymbol{f}\left(\boldsymbol{x}^{\prime}\right)\right)\right)
\end{aligned}
$$

with $\boldsymbol{F}$ the model matrix: $F_{i, j}=f_{i}\left(\boldsymbol{x}^{j}\right)$ and $\boldsymbol{\Psi}$ the correlation matrix: $\boldsymbol{\Psi}_{i, j}=$ $\psi\left(\mathbf{x}^{\mathbf{i}}, \mathbf{x}^{\mathbf{j}}\right), \boldsymbol{\psi}\left(\boldsymbol{x}^{\prime}\right)=\left[\psi\left(\boldsymbol{x}^{1}, \boldsymbol{x}^{\prime}\right), . ., \psi\left(\boldsymbol{x}^{n}, \boldsymbol{x}^{\prime}\right)\right]$ and the MLE of the coefficient vector and the process variance defined by: $\boldsymbol{\beta}=\left(\mathbf{F} \boldsymbol{\Psi}^{-1} \mathbf{F}\right)^{-1} \mathbf{F}^{T} \boldsymbol{\Psi}^{-1} \mathbf{y}$ and $\sigma^{2}=\frac{1}{n}(\boldsymbol{y}-\mathbf{F} \boldsymbol{\beta})^{T} \boldsymbol{\Psi}^{-1}(\boldsymbol{y}-\mathbf{F} \boldsymbol{\beta})$. Furthermore, an analytic expression of the partial derivatives of the surrogate to its input parameters can be derived [10, 11], given by:

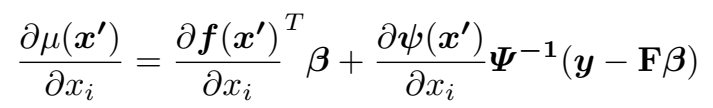

The idea of the gradient predictor is related to gradient-enhanced Kriging [10, 11]. But, in contrast to the aforementioned model, here the correlation function has to be only differentiable once since we do not need the second derivative of the correlation function to build the covariance matrix.

The accuracy of the surrogate can be significantly enhanced for the same computational budget if multiple fidelity models or grid levels are available [7, $15,33]$. Co-Kriging can be considered a powerful correction process which uses the correlation between cheap and expensive data to enhance the prediction accuracy. We refer to the most accurate expensive data values $\mathbf{y}_{\mathbf{e}}$ at points $\mathbf{X}_{\mathbf{e}}$ and the less accurate cheap data $\mathbf{y}_{\mathbf{c}}$ at points $\mathbf{X}_{\mathbf{c}}$. Conform the notion of correction processes, the co-Kriging formulation presents the surrogate of the expensive model $\mathbf{Y}_{\mathbf{e}}$ as the sum of the surrogate $\mathbf{Y}_{\mathbf{c}}$, of the cheap data $\left(\mathbf{X}_{\mathbf{c}}, \mathbf{y}_{\mathbf{c}}\right)$ 
and the surrogate $\mathbf{Y}_{\mathbf{d}}$, of the residuals $\left(\mathbf{X}_{\mathbf{e}}, \mathbf{y}_{\mathbf{e}}-\rho_{\mathbf{d}} \mu_{\mathbf{c}}\left(\mathbf{X}_{\mathbf{e}}\right)\right)$, with $\rho_{d}$ the scaling factor. The construction of the model occurs similar to the manner by which it was defined above: with a sequential construction of the two surrogates, where the scaling factor is determined through MLE along with the second surrogate.

The filtering of noise (e.g. due to discretization errors) can be achieved through the introduction of a regression constant $\lambda$ that is added to the diagonal of the covariance matrix $\boldsymbol{\Psi}[6]$. Consequently, the data is not interpolated as for $\left|\mathbf{x}^{(\mathbf{i})}-\mathbf{x}\right| \rightarrow \mathbf{0}$, the correlation becomes $\operatorname{cor}\left(\mathbf{x}^{(\mathbf{i})}, \mathbf{x}\right)=\mathbf{1}+\lambda$. The regression constant $\lambda$ is determined using maximum likelihood estimation in a similar manner as the other model parameters.

The construction of the Kriging model is performed using an open-source toolbox ooDACE (object-orientated Design and Analysis of Computer Experiments) [3]. The maximization of the concentrated log likelihood function is performed through a multi-start sequential quadratic programming methodology.

The stochastic nature of Kriging allows for the assessment of the uncertainty in the prediction. This can be used to define the expected improvement, which balances exploration (minimization of the uncertainty in the prediction) and exploitation (minimization of the objective). This infill criteria forms the basis of the well known efficient global optimization (EGO) algorithm by Jones et al. [13]. Keane et al. presented a multi-objective formulation of the expected improvement for two objectives based on the Euclidean distance and often referred to as such [14].

When dealing with a constrained optimization problem EI should decrease to zero when the constraint is violated. Given the surrogate of the constraint, we can calculate the probability of the prediction not violating the constraint limit, i.e. the probability that the constraint is met, $P[F(\boldsymbol{x})]$, where $F$ is the measure of feasibility. Under the assumption of uncorrelated objectives and constraints, it is straightforward to reformulate the expected improvement such that it accounts for the probability of feasibility $\mathbb{E}[I(\boldsymbol{x}) \cap F(\boldsymbol{x})]=$ $\mathbb{E}[I(\boldsymbol{x})] P[F(\boldsymbol{x})]$. This implies that at a given point in the design space, while the predicted constraint might be violated, the predicted errors in the constraint models are different from zero and as such the expectation of improvement will be low, but not zero, since there is a finite possibility that a full evaluation of the constraints may actually reveal a feasible design. This allows design space exploration in the early stages of the optimization methodology, but ensures convergence to the exact constrained optimum $[8,30,39]$.

\subsection{Optimization Framework}

Combining all the aforementioned, we can create a surrogate based optimization methodology (Figure 3). First, a LHS of $10 d$ points (with $d$ the dimensionality of the problem, equal to $6: 4$ design parameters and 2 operating parameters, $\alpha$ and $\delta$ ) is created to be evaluated by the low fidelity model and from 
this LHS a space-filling subset of $3 d$ points is selected to be evaluated by the high fidelity model. The selection of samples follows the guidelines from Toal et al. [33], Loeppky et al. [19] and Marrel et al. [20]. Therefore it is expected that the influence of another LHS on the outcome of the optimization would be small. However, this was not verified due to the high computational cost of the optimization. Furthermore, the boundariescornerpoints of the design space are also evaluated by both the low and high fidelity models.

The evaluation of the design sets through URANS simulations using the $\gamma-R e_{\theta}$ model performed at a fixed velocity ${ }^{2}$ close to the stall speed gives us $C_{L}(\mathbf{x}), C_{D}(\mathbf{x}), C_{M, p i t c h}(\mathbf{x})$ and $C_{M, \text { roll }}(\mathbf{x})$. The former two can be plugged in the horizontal and vertical equilibrium equations to determine the minimum $V$ along with the corresponding $T$.

From the obtained $V_{\text {min }}(\mathbf{x}), C_{M, p i t c h}(\mathbf{x})$ and $C_{M, \text { roll }}(\mathbf{x})$, surrogates can be constructed that are cheap to evaluate and can produce the partial derivatives to the different design parameters. This allows the evaluation of the objectives for every parameter set combination. Furthermore, two constraints can be evaluated: $P\left[C_{M, p i t c h}(\boldsymbol{x})=0\right]$ which corresponds to the momentum equilibrium equation and $P\left[\partial C_{M, p i t c h}(\boldsymbol{x}) / \partial \alpha<0\right]$ which corresponds to longitudinal static stability, and thus absence of the nose-up pitching moment attributed to tip stall. Through the introduction of surrogates, which allow a direct analytic evaluation of their gradients, we avoid the need to perform adjoint or costly finite difference approaches to determine both the second objective and constraint, which leads to a very efficient optimization framework.

The error in the prediction attributed to the descent speed is directly obtained from the surrogate. However, for the controllability coefficient it is calculated indirectly through the use of error propagation formulas:

$$
s_{M^{\prime}}^{2}\left(\boldsymbol{x}^{\prime}\right)=M^{\prime}\left(\boldsymbol{x}^{\prime}\right)^{2}\left[\left(\frac{s_{c_{M, \text { roll }}}\left(\boldsymbol{x}^{\prime}\right)}{c_{M, \text { roll }}\left(\boldsymbol{x}^{\prime}\right)}\right)^{2}+\left(\frac{2 V\left(\boldsymbol{x}^{\prime}\right) s_{V}\left(\boldsymbol{x}^{\prime}\right)}{V\left(\boldsymbol{x}^{\prime}\right)^{2}}\right)^{2}\right]
$$

with $s_{c_{M, \text { roll }}}$ and $s_{V}$ respectively the standard deviation of the prediction of the surrogate of the roll moment coefficient $c_{M \text {,roll }}$ and the velocity $s_{V}$.

While this introduces a correlation between the objective functions, we further assume them to be uncorrelated and use the multi-objective expected improvement formula multiplied with the two constraints. To determine the next infill point, we maximize this MOEI with a multistart SQP algorithm to ensure a global optimum of the multi-model objective, which is then evaluated using the high fidelity model. This optimization procedure is then repeated until convergence, $E I_{n o r m}{ }^{3}<1 \%$, or until the calculation budget runs out, corresponding to an additional 40 calculation, corresponding with an infill to DoE ratio of roughly $1: 2$.

\footnotetext{
2 We assume at this point that the variations in $R e$ during the optimization are small enough to be negligible

3 The constrained expected improvement is divided by the Euclidean distance of the two points farthest from each other in de objective space.
} 
Conventional optimization techniques that optimize a design for a number of operation conditions (multipoint) take a weighted sum of their objective function $[24,16]$. However, this only moves the problem further downstream: what weight is attributed to which condition? Furthermore, operation conditions such as stall are not known in advance and should be determined iteratively. By adding the operations variables to the optimization problem, the aforementioned limitations can be overcome. The continuity of alpha and delta results in a continuous optimization The continuity of $\alpha$ and $\delta$ results in a continuous optimization. Thus, changing $\alpha$ for every fence design results in a new $\delta$ (to obtain $C_{M, \text { pitch }}=0$ ), $C_{L}$ and $C_{D}$ and consequently a changing $V$ and $M^{\prime}$. Every fence introduces thus by itself a Pareto front ${ }^{4}$ : decreasing the $\alpha$ leads to a higher $V$ and higher $M^{\prime}$. The Pareto front of the entire design space is thus a summation of Pareto fronts of fences.

\section{Results and Discussion}

\subsection{Optimization Convergence}

To illustrate the convergence of the optimization methodology, the first six iterations after the DoE are displayed below (figure 4). In blue we see the unconstrained predicted Pareto front, determined by the objective functions. The red line represents the constraint predicted Pareto front, of which the design are characterized by $C_{M, p i t c h}=0$ and $\partial C_{M, p i t c h} / \partial \alpha<0$. The black line represents the initial design, noted for its complete absence of a fence. The green line represents the Pareto front made up out of evaluated points that meet the constraints. It is the objective to bring the latter as close as possible to the predicted constrained Pareto front. The light blue dots indicate the Pareto front of evaluated points which are either characterized by $C_{M, \text { pitch }} \neq 0$ and/or $\partial C_{M, p i t c h} \partial \alpha>0$. The purple dot presents the next infill point with maximum constrained MOEI. All the predicted Pareto fronts are found using NSGA-ii.

A first observation that can be drawn when comparing the first six iterations and the final Pareto front (figure 4g) is how the unconstrained Pareto front shows little movement. This indicates that the surrogates of velocity and controllability are already fairly accurate after the DoE. Secondly, the constrained Pareto front shows strong movement, indicating the early inaccuracy of the pitching moment surrogate. This can also be derived from the fact that the evaluated Pareto front does not change in the first four iterations, indicating that the new infill points did not meet the constraints. A third observation to be drawn is how the evaluated points move around. This is caused by the fact that the controllability coefficient is obtained indirectly through the surrogate as opposed to the velocity.

4 The Pareto front is the front defined in the objective space by the Pareto optimal points for which one cannot improve on one without deteriorating on the others. 
Fig. 3: Flowchart of the optimization framework

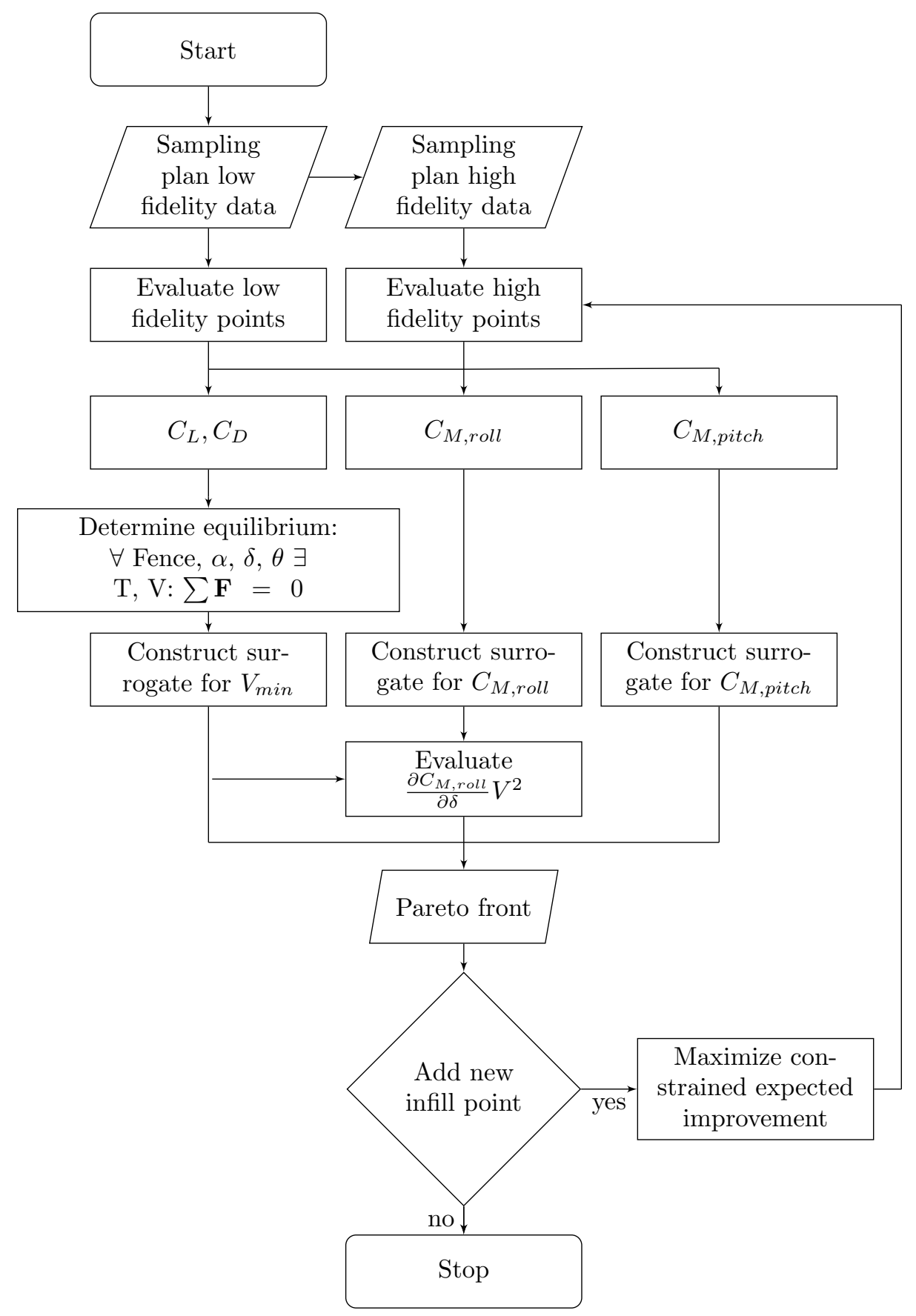


To illustrate the convergence of the optimization methodology, the change of the normalized hypervolumes ${ }^{56}$ of the unconstrained, constrained and clean wing Pareto front after the DoE are illustrated below along with the stopping criterion (Figure 4h). The predicted Pareto fronts are presented here instead of the evaluated onces because we are using a regression model under the assumption that with the addition of infills the surrogate will become correcter than the CFD simulations, which are subjected to a discretization error. It can be observed that the unconstrained Pareto fronts and the initial design stay fairly constant during the optimization. This is conform earlier observations. Furthermore, the inital design stabilized at iteration 15 and the unconstrained Pareto front stabilizes at iteration 30, at which point the expected improvement decreases to nearly zero. On the other hand, the constrained Pareto front upholds a fluctuating behavior, indicating that the surrogate of the pitching moment is still moving around and that additional infill points are required. This indicates that caution is in order when using the normalized constrained expected improvement as stopping criterion.

\subsection{Optimal Fences: Pareto Front}

A first observation that can be drawn when examining the Pareto fronts after the final iteration (figure $4 \mathrm{~g}$ ) is the limited decrease in the descent speed that can be obtained, while on the other hand a noteworthy increase of controllability is more attainable. The limited speed gain corresponds to the findings of Das [31]. Haines attributed the significant lift increase attainable to the presence of a leading edge vortex [9], which is absent altogether on the UAV examined here.

An assessment of three different fence geometries that have been evaluated and meet the constraints is presented below: on one end of the Pareto front, the fence with which the lowest descent speed is obtainable, to which we will refer as minimal speed design (MSD), on the other end of the Pareto front, the fence with which the highest controllability is obtainable, to which we will refer as the maximal controllability design (MCD), and a fence which outperforms both objectives in comparison with the initial design, to which we will refer as the overall better design (OBD). Since every fence design, and thus also the absence of a fence, leads to a Pareto front, we refer to the condition of maximum controllability in the absence of a fence as the maximal controllability initial design (MCID) and the condition of minimum descent speed as the minimal speed initial design (MSID). The parameters of the respective designs are presented in table 1 and figure 5 .

\footnotetext{
5 The hyperholume is the Lebesgue measure contained by the attainment surface and a chosen reference points. The attainment surface was defined by Fonseca \& Fleming [5] as "the boundary in the objective space separating those points which are dominated by or equal to at least one of the data points, from those which no data points dominates or equals" and thus corresponds to the Pareto front.

6 The Pareto fronts are rescaled to a 1-on-1 box. This is done such that the influence of both objectives on the hypervolume is nearly equally significant.
} 


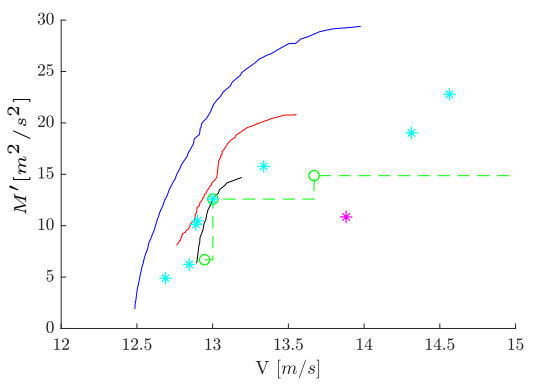

(a) $1^{\text {st }}$ iteration

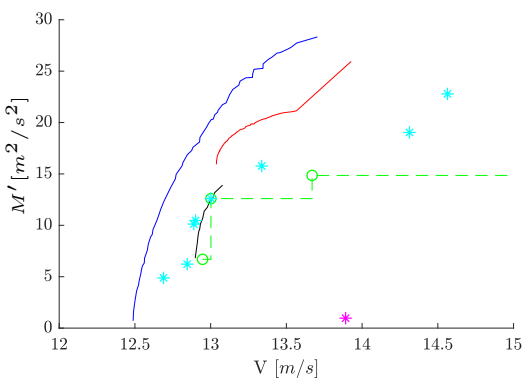

(c) $3^{\text {rd }}$ iteration

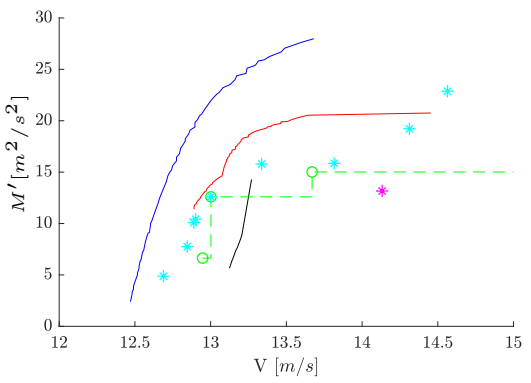

(e) $5^{\text {th }}$ iteration

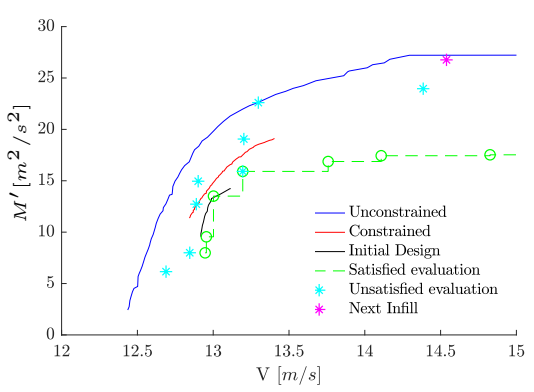

(g) Final iteration

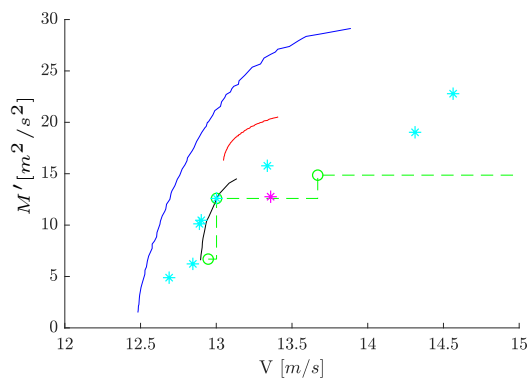

(b) $2^{\text {nd }}$ iteration

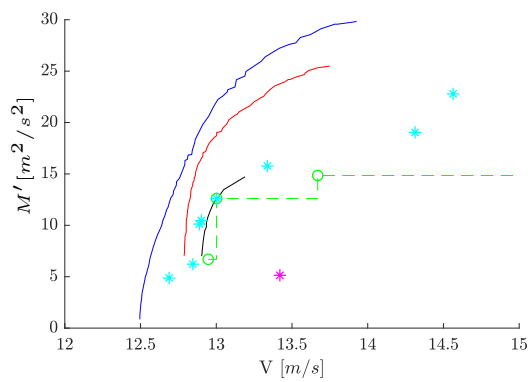

(d) $4^{\text {th }}$ iteration

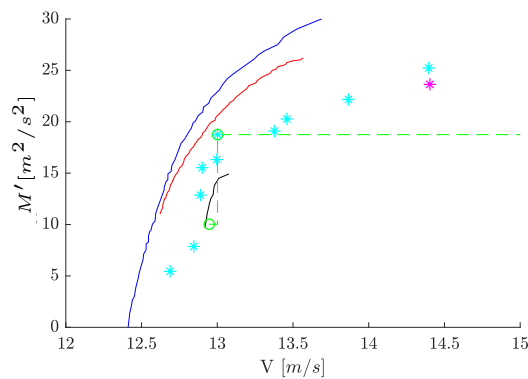

(f) $6^{\text {th }}$ iteration

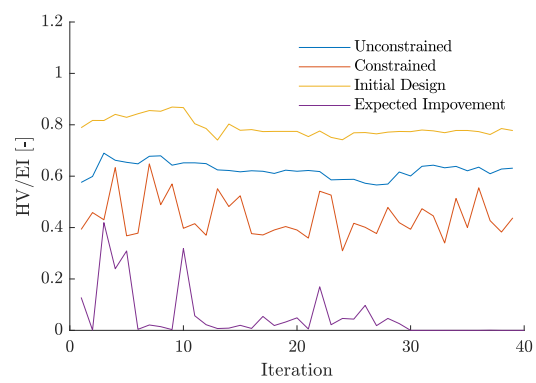

(h) Metric convergence

Fig. 4: Convergence of the optimization algorithm. 
Moving along the constrained predicted Pareto front from minimal speed to maximal controllability, a decrease of angle of attack is to be observed, along with an increase of elevon deflection, inward movement (away from the tip) of the fence, a decrease of the height and both an increase of length on suction and pressure side. The decrease of $\alpha$ leads to a lower $C_{L}$ and thus higher $V$ and higher $M^{\prime}$. The decrease of $s$ leads to a bigger region of attached flow at the tip, thus requires a larger $\delta$ to uphold pitching equilibrium, which in turn reduces $C_{L}$ but increases the effectiveness of the elevons and thus increases $M^{\prime}$. A decrease of the length on the suction side requires an increase of the height to maintain the same effectiveness. A shorter fence length on the suction side leads to a later separation on the inboard side and a higher $C_{L}$ thus lower $V$ and corresponding lower $M^{\prime}$. An increase of the length of the fence on the pressure side leads to a redirection of the flow on the pressure side towards the elevon and increases its effectiveness, thus increasing $M^{\prime}$.

Table 1: Optimal fence designs

\begin{tabular}{c|cccccc|cc} 
Design & $\begin{array}{c}\alpha \\
\left.{ }^{o}\right]\end{array}$ & $\begin{array}{c}\delta \\
\left.{ }^{\circ}\right]\end{array}$ & $\begin{array}{c}s \\
{[\%]}\end{array}$ & $\begin{array}{c}h \\
{[\%]}\end{array}$ & $\begin{array}{c}l_{\text {up }} \\
{[\%]}\end{array}$ & $\begin{array}{c}l_{\text {down }} \\
{[\%]}\end{array}$ & $\begin{array}{c}V \\
{[\mathrm{~m} / \mathrm{s}]}\end{array}$ & $\begin{array}{c}M^{\prime} \\
{\left[\mathrm{m}^{2} / \mathrm{s}^{2}\right]}\end{array}$ \\
\hline \hline MSID & 14.6 & 3.607 & - & - & - & - & 13.58 & 7.4186 \\
MCID & 10.0 & 3.056 & - & - & - & - & 18.32 & 10.00 \\
\hline MSD & 15.445 & 3.662 & 54.30 & 80.43 & 5.45 & 5.19 & 12.59 & 8.09 \\
OBD & 15.185 & 5.008 & 48.85 & 79.13 & 7.13 & 13.50 & 12.87 & 13.36 \\
MCD & 14.856 & 6.215 & 47.82 & 74.52 & 11.04 & 85.54 & 13.70 & 20.16
\end{tabular}

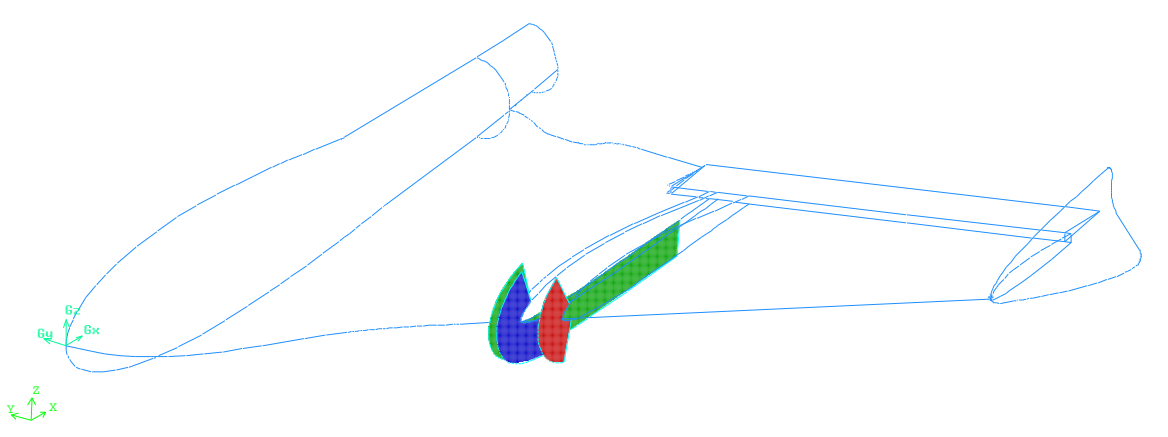

Fig. 5: Optimal fence designs (MSD in red, OBD in blue and MCD in green) 


\section{Conclusion}

In this paper a multi-objective multi-fidelity surrogate based optimization of the stall characteristics of an unmanned aerial vehicle through the addition of a wing fence was performed. The UAV under consideration is characterized by the appearance of tip stall, leading to a nose up pitching moment and loss of control. The introduction of a wing fence, a small plate placed on top of the wing aligned with the flow, alters the stall behavior by moving the point of separation away from the tip to the inboard side of the fence. With the objective of minimizing the descent speed and maximizing the controllability, defined as the roll moment initiated through an elevon deflection around its pitching moment equilibrium, the wing fence, parameterized by location, height and length on both pressure and suction side, was adapted. Furthermore, the angle of attack and elevon deflection were added as parameters, resulting in a continuous optimization. In addition to the objectives, a constraint to obtain the pitching moment equilibrium and a constraint to ensure longitudinal static stability, which goes hand in hand with the absence of tip stall, were introduced.

A framework was built around regressive universal co-Kriging combining two fidelity levels based on grid size and using regression rather than interpolation to account for the appearance of discretization and averaging errors. By building surrogates for both the objectives and constraints, a trustworthy, but relatively inexpensive optimization was obtained. The constraint of stability and objective of controllability correspond to gradients from CFD output and are calculated analytically using the surrogates, effectively avoiding the need to determining the gradients using CFD which can be both hard (using adjoints) or costly (using finite difference schemes). Assessment of the hypervolumes and infill criterion convergence has shown the importance of defining a correct stopping criterion when dealing with constrained problems using surrogate-derived gradients.

The result is a Pareto front of fence designs and angle of attack and elevon settings that may outperform the initial design in either obtaining a slower descent speed or higher controllability or both.

\section{Acknowledgments}

The authors would like to thank prof. dr. ir. Jan Vierendeels; his input, supervision, guidance and support during this research has been of critical value.

\section{Conflict of interest}

On behalf of all authors, the corresponding author states that there is no conflict of interest. 


\section{Replication of Results}

The framework, written in bash, linking together mesh generation (Gambit), simulation (ANSYS Fluent) and optimization (Matlab), that performs the grid convergence study and optimization has been made available as supplementary material on the SMDO website.

\section{References}

1. Bevan, R.L.T., Poole, D.J., Allen, C.B., Rendall, T.C.S.: Adaptive surrogate-based optimization of vortex generators for tiltrotor geometry. Journal of Aircraft 54(3), 10111024 (2017). DOI 10.2514/1.C033838

2. Couckuyt, I., Deschrijver, D., Dhaene, T.: Fast calculation of multiobjective probability of improvement and expected improvement criteria for pareto optimization. Journal of Global Optimization 60(3), 575-594 (2014). DOI 10.1007/s10898-013-0118-2

3. Couckuyt, I., Dhaene, T., Demeester, P.: oodace toolbox: a flexible object-oriented kriging implementation. Journal of Machine Learning Research 15(1), 3183-3186 (2014)

4. Dhamotharan, V., Jadhav, P.D., Ramu, P., Prakash, A.K.: Optimal design of savonius wind turbines using ensemble of surrogates and cfd analysis. Structural and Multidisciplinary Optimization 58(6), 2711-2726 (2018). DOI 10.1007/s00158-018-2052-x

5. Fonseca, C.M., Fleming, P.J.: On the performance assessment and comparison of stochastic multiobjective optimizers. In: H.M. Voigt, W. Ebeling, I. Rechenberg, H.P. Schwefel (eds.) Parallel Problem Solving from Nature - PPSN IV, pp. 584-593. Springer Berlin Heidelberg (1996)

6. Forrester, A., Sóbester, A., Keane, A.: Optimization with missing data. Proceedings of the Royal Society A: Mathematical, Physical and Engineering Science 462(2067), 935 (2006). DOI 10.1098/rspa.2005.1608

7. Forrester, A., Sóbester, A., Keane, A.: Multi-fidelity optimization via surrogate modelling. Proceedings of the Royal Society a-Mathematical Physical and Engineering Sciences 463(2088), 3251-3269 (2007). DOI 10.1098/rspa.2007.1900

8. Forrester, A., Sóbester, A., Keane, A.: Engineering Design via Surrogate Modelling: A Practical Guide. Wiley (2008)

9. Haines, A.B.: Aerodynamic Interference. AGARD Special Course on Subsonic/Transonic Aerodynamic Interference for Aircraft. (1980)

10. Han, Z.H., Görtz, S., Zimmermann, R.: Improving variable-fidelity surrogate modeling via gradient-enhanced kriging and a generalized hybrid bridge function. Aerospace Science and Technology 25(1), 177-189 (2013). DOI 10.1016/j.ast.2012.01.006

11. Han, Z.H., Zhang, Y., Song, C.X., Zhang, K.S.: Weighted gradient-enhanced kriging for high-dimensional surrogate modeling and design optimization. AIAA Journal 55(12), 4330-4346 (2017). DOI 10.2514/1.J055842

12. Jeong, S., Obayashi, S.: Efficient global optimization (EGO) for multi-objective problem and data mining. In: 2005 IEEE Congress on Evolutionary Computation, vol. 3, pp. 2138-2145 Vol. 3 (2005). DOI 10.1109/CEC.2005.1554959

13. Jones, D.R., Schonlau, M., Welch, W.J.: Efficient global optimization of expensive black-box functions. Journal of Global Optimization 13(4), 455-492 (1998). DOI 10.1023/A:1008306431147

14. Keane, A.J.: Statistical improvement criteria for use in multiobjective design optimization. AIAA Journal 44(4), 879-891 (2006). DOI 10.2514/1.16875

15. Kennedy, M.C., O'Hagan, A.: Predicting the output from a complex computer code when fast approximations are available. Biometrika 87(1), 1-13 (2000). DOI 10.1093/biomet/87.1.1

16. Kenway, G.K.W., Martins, J.R.R.A.: Multipoint aerodynamic shape optimization investigations of the common research model wing. AIAA Journal 54(1), 113-128 (2015). DOI 10.2514/1.J054154 
17. Knowles, J.: Parego: A hybrid algorithm with on-line landscape approximation for expensive multiobjective optimization problems. IEEE Transactions on Evolutionary Computation 10(1), 50-66 (2005). DOI 10.1109/TEVC.2005.851274

18. Lissaman, P.B.S.: Low-Reynolds-number airfoils. Annual Review of Fluid Mechanics 15(1), 223-239 (1983). DOI 10.1146/annurev.fl.15.010183.001255

19. Loeppky, J.L., Sacks, J., Welch, W.J.: Choosing the sample size of a computer experiment: A practical guide. Technometrics 51(4), 366-376 (2009). DOI 10.1198/TECH.2009.08040

20. Marrel, A., Iooss, B., Laurent, B., Roustant, O.: Calculations of sobol indices for the gaussian process metamodel. Reliability Engineering and System Safety 94(3), 742-751 (2009). DOI 101016/jress200807008

21. McKay, M.D., Beckman, R.J., Conover, W.J.: A comparison of three methods for selecting values of input variables in the analysis of output from a computer code. Technometrics 21(2), 239-245 (1979). DOI 10.2307/1268522

22. Menter, F.R., Langtry, R.B., Likki, S.R., Suzen, Y.B., Huang, P.G., Volker, S.: A correlation-based transition model using local variables - part I: Model formulation. Journal of Turbomachinery-Transactions of the Asme 128(3), 413-422 (2006). DOI $10.1115 / 1.2184352$

23. Morris, M.D., Mitchell, T.J.: Exploratory designs for computational experiments. Journal of Statistical Planning and Inference 43(3), 381-402 (1995). DOI 10.1016/03783758(94)00035-T

24. Namura, N., Obayashi, S., Jeong, S.: Efficient global optimization of vortex generators on a supercritical infinite wing. Journal of Aircraft 53(6), 1670-1679 (2016). DOI 10.2514/1.C033753

25. Nickel, K., Wohlfahrt, M.: Tailless aircraft in theory and practice. American Institute of Aeronautics and Astronautics, Washington, DC (1994)

26. Okonkwo, P., Smith, H.: Review of evolving trends in blended wing body aircraft design. Progress in Aerospace Sciences 82, 1-23 (2016). DOI 10.1016/j.paerosci.2015.12.002

27. Perry, D., Port, W.: Low speed flight tests on a tailless delta wing aircraft (avro 707b): Part 4 - wing flow. Report ARC-CP-1107 (1970)

28. Ponweiser, W., Wagner, T., Biermann, D., Vincze, M.: Multiobjective optimization on a limited budget of evaluations using model-assisted $\mathcal{S}$-metric selection. In: G. Rudolph, T. Jansen, N. Beume, S. Lucas, C. Poloni (eds.) Parallel Problem Solving from Nature - PPSN X, pp. 784-794. Springer Berlin Heidelberg

29. Roache, P.J.: Perspective: A method for uniform reporting of grid refinement studies. Journal of Fluids Engineering 116(3), 405-413 (1994). DOI 10.1115/1.2910291

30. Sasena, M., Papalambros, P., Goovaerts, P.: Exploration of meta-modeling sampling criteria for constrained global optimization. Engineering Optimization 34, 263-278 (2002)

31. Schlichting, V.H.: Einige Neuere Ergebnisse Über Grenzschichtbeeinflussung: Recent Developments in Boundary Layer Control, pp. 563-586. Pergamon (1959). DOI 10.1016/B978-1-4831-9833-0.50005-0

32. Shi, R., Liu, L., Long, T., Wu, Y., Wang, G.G.: Multidisciplinary modeling and surrogate assisted optimization for satellite constellation systems. Structural and Multidisciplinary Optimization (5), 2173-2188 (2018). DOI 10.1007/s00158-018-2032-1

33. Toal, D.J.J.: Some considerations regarding the use of multi-fidelity kriging in the construction of surrogate models. Structural and Multidisciplinary Optimization 51(6), 1223-1245 (2015). DOI 10.1007/s00158-014-1209-5. URL https://doi.org/10.1007/s00158-014-1209-5

34. Toal, D.J.J.: A study into the potential of gpus for the efficient construction and evaluation of kriging models. Engineering with Computers 32(3), 377-404 (2016). DOI 10.1007/s00366-015-0421-2. URL https://doi.org/10.1007/s00366-015-0421-2

35. Wauters, J., Degroote, J.: On the study of transitional low-reynolds number flows over airfoils operating at high angles of attack and their prediction using transitional turbulence models. Progress in Aerospace Sciences 103, 52 - 68 (2018). DOI 10.1016/j.paerosci.2018.10.004

36. Wauters, J., Degroote, J., Vierendeels, J.: Comparative study of transition models for high-angle-of-attack behavior. AIAA Journal pp. 1-16 (2019). DOI 10.2514/1.J057249 
37. Williams, M.D., Reeder, M.F., Maple, R.C., Solfelt, D.A.: Modeling, simulation, and flight tests for a t-38 talon with wing fences. Journal of Aircraft 47(2), 423-433 (2010). DOI $10.2514 / 1.46122$

38. Wu, X., Zhang, W., Song, S.: Robust aerodynamic shape design based on an adaptive stochastic optimization framework. Structural and Multidisciplinary Optimization 57(2), 639-651 (2018). DOI 10.1007/s00158-017-1766-5

39. Zhang, K.S., Han, Z.H., Gao, Z.J., Wang, Y.: Constraint aggregation for large number of constraints in wing surrogate-based optimization. Structural and Multidisciplinary Optimization 59(2), 421-438 (2019). DOI 10.1007/s00158-018-2074-4. URL $10.1007 / \mathrm{s} 00158-018-2074-4$

40. Zhang, Q., Liu, W., Tsang, E., Virginas, B.: Expensive multiobjective optimization by moea/d with gaussian process model. IEEE Transactions on Evolutionary Computation 14(3), 456-474 (2010). DOI 10.1109/TEVC.2009.2033671 